\title{
Research Article \\ Global Stability for a Binge Drinking Model with Two Stages
}

\author{
Hai-Feng Huo and Na-Na Song \\ Department of Applied Mathematics, Lanzhou University of Technology, Gansu, Lanzhou 730050, China \\ Correspondence should be addressed to Hai-Feng Huo, huohf70@sohu.com
}

Received 18 July 2012; Revised 6 September 2012; Accepted 4 October 2012

Academic Editor: Seenith Sivasundaram

Copyright (C) 2012 H.-F. Huo and N.-N. Song. This is an open access article distributed under the Creative Commons Attribution License, which permits unrestricted use, distribution, and reproduction in any medium, provided the original work is properly cited.

A more realistic two-stage model for binge drinking problem is introduced, where the youths with alcohol problems are divided into those who admit the problem and those who do not admit it. We also consider the direct transfer from the class of susceptible individuals towards the class of admitting drinkers. Mathematical analyses establish that the global dynamics of the model are determined by the basic reproduction number, $R_{0}$. The alcohol-free equilibrium is globally asymptotically stable, and the alcohol problems are eliminated from the population if $R_{0}<1$. A unique alcohol-present equilibrium is globally asymptotically stable if $R_{0}>1$. Numerical simulations are also conducted in the analytic results.

\section{Introduction}

Young people's binge drinking problem is a major concern to public health. Recently, US surveys indicate that approximately $90 \%$ of college students have consumed alcohol at least once [1], and more than $40 \%$ of college students have engaged in binge drinking $[2,3]$. The binge drinking refers to youths in 17-30 age group who drink a large amount of alcohol and become so drunk; they are likely to exhibit antisocial behavior [4]. Although there have been many attempts to reduce the problem, alcohol abuse by college students has persisted and in some cases increased over the past several decades [5]. Prior studies have indicated that heavy alcohol drinkers are likely to engage in risky sexual behaviours and more likely to get sexually transmitted infection than social drinkers $[6,7]$. There is a strong medical evidence that treatment of individuals with alcohol problems is a major issue [8-10].

Thus, it is very important to use a mathematical method to study the binge drinking problems in youths. A simple model for alcohol treatment is presented by Sanchez et al. [11]. Since then, there have been numerous studies investigating campus drinking and 
the associated consequences [12-15]. Manthey et al. [12] focus on a college campus, divide the student population into three classes: nondrinkers, social drinkers and problem drinkers, and show that campus alcohol abuse may be reduced by minimizing the ability of problem drinkers to directly recruit nondrinkers. Cintron-Arias et al. [13] focus on situations where relapse rates are high and conclude that the systematic removal of individuals from highrisk environments, or the development of programs that limit access or reduce the residence times in such environments (or both approaches combined), may reduce the level of alcohol abuse. Mubayi et al. [14] show that if the relative residence times of moderate drinkers are distributed randomly between low- and high-risk environments, then the proportion of heavy drinkers is likely to be higher than expected. Mulone and Straughan [15] investigate a model for binge drinking taking into account admitting and nonadmitting drinkers. But the global stability of binge drinking model is not discussed in the literature.

Motivated by the binge drinking model in [15], we develop a more realistic model with two stages. Drinking is often encouraged by peer pressure. A susceptible individual acquires alcohol problems through the direct contact with the admitting drinker or the nonadmitting drinker. The new drinker can become either the admitting drinker or the nonadmitting drinker. So we consider the direct transfer from the class of susceptible individuals towards the class of admitting drinkers; furthermore, we study the global dynamics of the model. The reason to introduce this new direct transfer is that about one-third of the American population admit to drinking problems, $17.8 \%$ of the population admit to the binge drinking problem [16]. Therefore, this fact cannot be neglected in the binge drinking model.

The organization of this paper is as follows. In the next section, the binge drinking model with two stages and some basic properties are derived. In Section 3, the existence and the global stability of equilibria are investigated. Some numerical simulations are given in Section 4. Some discussions are given in Section 5.

\section{The Model}

\subsection{System Description}

The total population is divided into four compartments, namely, the susceptible compartment of those who do not drink or drink only moderately, denoted by $S(t)$, those who drink heavily at least some of the time but do not admit having a problem, denoted by $A_{1}(t)$, those who drink heavily and admit having a problem, denoted by $A_{2}(t)$, and those people in treatment, denoted by $R(t)$. The total number of population at time $t$ is given by

$$
N(t)=S(t)+A_{1}(t)+A_{2}(t)+R(t)
$$

The model structure is shown in Figure 1. The transfer diagram leads to the following system of ordinary differential equations:

$$
\begin{gathered}
\dot{S}=\mu N-\frac{S\left(\beta A_{1}+\gamma A_{2}\right)}{N}-\mu S, \\
\dot{A}_{1}=\frac{(1-p) S\left(\beta A_{1}+\gamma A_{2}\right)}{N}-(\alpha+\mu) A_{1},
\end{gathered}
$$




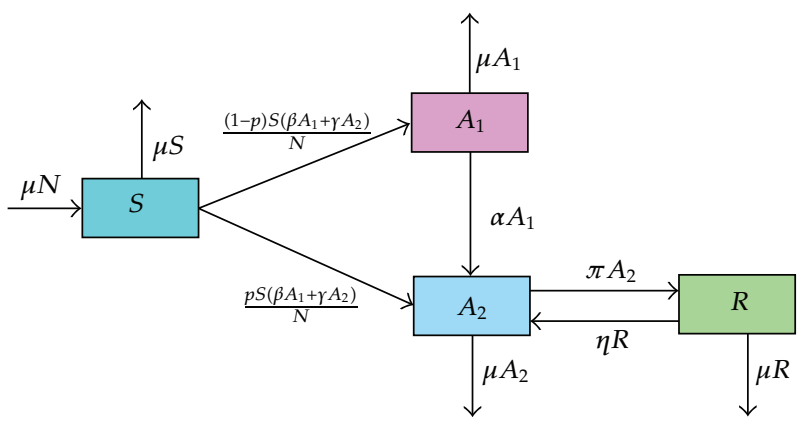

Figure 1: Transfer diagram of model (2.2).

$$
\begin{gathered}
\dot{A}_{2}=\frac{p S\left(\beta A_{1}+\gamma A_{2}\right)}{N}+\eta R+\alpha A_{1}-(\pi+\mu) A_{2}, \\
\dot{R}=\pi A_{2}-(\mu+\eta) R,
\end{gathered}
$$

where $\mu N$ is the number of individuals entering into the system in a given time interval (say each year), so $\mu$ represents the rate of entry. Since we are dealing with youths, we assume that the death rate is negligible, and so the leaving rate is also $\mu$. A susceptible individual can be turned to drink through direct contact with an admitting drinker or a nonadmitting drinker. $\beta$ is the transmission coefficient for the individuals who do not admit to have alcohol problems, $\gamma$ is the transmission coefficient for the individuals who admit to have these problems. We assume that $\beta>\gamma$ due to the fact that they are more likely to be unaware of their condition. $\alpha$ is the rate at which represents those with the alcohol problems admitting to have these problems and then transferring from class $A_{1}$ to $A_{2}, \pi$ is that fraction of $A_{2}$ who go into treatment, $\eta$ is that fraction of $R$ who relapse into admitting drinkers (we adopt a linear relapse term rather than peer pressure since we argue that the relapse is primarily due to the person), $p$ is the probability of a new drinker to admit having the problem. We may show that $N=S+A_{1}+A_{2}+R$ is constant, and then we introduce the fractions of $S, A_{1}, A_{2}$, and $R$ :

$$
s=\frac{S}{N}, \quad a_{1}=\frac{A_{1}}{N}, \quad a_{2}=\frac{A_{2}}{N}, \quad r=\frac{R}{N},
$$

with $s+a_{1}+a_{2}+r=1$. Then the system (2.2) becomes

$$
\begin{gathered}
\dot{s}=\mu-s\left(\beta a_{1}+\gamma a_{2}\right)-\mu s, \\
\dot{a}_{1}=(1-p) s\left(\beta a_{1}+\gamma a_{2}\right)-(\alpha+\mu) a_{1}, \\
\dot{a}_{2}=p s\left(\beta a_{1}+\gamma a_{2}\right)+\eta r+\alpha a_{1}-(\pi+\mu) a_{2}, \\
\dot{r}=\pi a_{2}-(\eta+\mu) r .
\end{gathered}
$$

As system (2.4) is equivalent to system (2.2), we only need to study system (2.4). 


\subsection{Basic Properties}

\subsubsection{Invariant Region}

Adding all the equations of system (2.4) gives

$$
\frac{d N}{d t}=0
$$

Thus, the total population $N$ is a constant. Since system (2.4) monitors human population, it is plausible to assume that all its state variables and parameters are nonnegative for all $t \geq 0$. Further, it can be shown that the region

$$
\Omega=\left\{\left(s(t), a_{1}(t), a_{2}(t), r(t)\right) \in R_{+}^{4}: s(t)+a_{1}(t)+a_{2}(t)+r(t) \leq 1\right\}
$$

is positively invariant. Thus, each solution of system (2.4), with initial conditions in $\Omega$, remains there for $t \geq 0$. Therefore, the $\omega$-limit sets of the solutions of system (2.4), in $\Omega$, are contained in $\Omega$. Furthermore, in $\Omega$, the usual existence, uniqueness, and continuation results hold for the system, so that the system (2.4) is well posed mathematically and epidemiologically [17]. So we consider the dynamics of system (2.4) on the set $\Omega$ in this paper.

\subsubsection{Positivity of Solutions}

For system (2.4), it is necessary to prove that all the state variables are positive, so that the solutions of the system with positive initial conditions remain positive for all $t \geq 0$. We thus state the following lemma.

Lemma 2.1. If $s(0)>0, a_{1}(0)>0, a_{2}(0)>0$, and $r(0)>0$, the solutions $s(t), a_{1}(t), a_{2}(t)$, and $r(t)$ of system (2.4) are positive for all $t \geq 0$.

Proof. Under the given initial conditions, it is easy to prove that the solutions of system (2.4) are positive; if not, we assume a contradiction: that there exists a first time $t_{1}$ such that

$$
s\left(t_{1}\right)=0, \quad s^{\prime}\left(t_{1}\right)<0, \quad a_{1}(t)>0, \quad a_{2}(t)>0, \quad r(t)>0, \quad 0<t<t_{1},
$$

there exists a $t_{2}$,

$$
a_{1}\left(t_{2}\right)=0, \quad a_{1}^{\prime}\left(t_{2}\right)<0, \quad s(t)>0, \quad a_{2}(t)>0, \quad r(t)>0, \quad 0<t<t_{2},
$$

there exists a $t_{3}$,

$$
a_{2}\left(t_{3}\right)=0, \quad a_{2}^{\prime}\left(t_{3}\right)<0, \quad s(t)>0, \quad a_{1}(t)>0, \quad r(t)>0, \quad 0<t<t_{3},
$$


and there exists a $t_{4}$

$$
r\left(t_{4}\right)=0, \quad r^{\prime}\left(t_{4}\right)<0, \quad s(t)>0, \quad a_{1}(t)>0, \quad a_{2}(t)>0, \quad 0<t<t_{4} .
$$

In the first case, we have

$$
s^{\prime}\left(t_{1}\right)=\mu>0,
$$

which is a contradiction, meaning that $s(t)>0, t \geq 0$. In the second case, we have

$$
a_{1}^{\prime}\left(t_{2}\right)=(1-p) \gamma a_{2}(t) s(t)>0,
$$

which is a contradiction, meaning that $a_{1}(t)>0, t \geq 0$. Similarly, it can be shown that $a_{2}(t)>0$ and $r(t)>0$ for all $t \geq 0$. $t>0$.

Thus, the solutions $s(t), a_{1}(t), a_{2}(t)$, and $r(t)$ of system (2.4) remain positive for all

\section{Analysis of the Model}

There are one alcohol-free equilibrium $E_{0}$ and one alcohol-present equilibrium $E^{*}$ for system (2.4).

\subsection{Alcohol-Free Equilibrium and the Reproduction Number}

The model has an alcohol-free equilibrium given by

$$
E_{0}=(1,0,0,0)
$$

In the following, the basic reproduction number of system (2.4) will be obtained by the nextgeneration matrix method formulated in [18].

Let $x=\left(a_{1}, a_{2}, r, s\right)^{T}$, then system (2.4) can be written as

$$
\frac{d x}{d t}=\mp(x)-\mathcal{U}(x)
$$


where

$$
\begin{gathered}
\mathcal{F}(x)=\left(\begin{array}{c}
(1-p)\left(\beta a_{1}+\gamma a_{2}\right) s \\
p\left(\beta a_{1}+\gamma a_{2}\right) s \\
0 \\
0
\end{array}\right), \\
\mho(x)=\left(\begin{array}{c}
(\alpha+\mu) a_{1} \\
\pi+\mu) a_{2}-\alpha a_{1}-\eta r \\
(\eta+\mu) r-\pi a_{2} \\
\mu s+\left(\beta a_{1}+\gamma a_{2}\right) s-\mu
\end{array}\right) .
\end{gathered}
$$

The Jacobian matrices of $\mathcal{F}(x)$ and $\mho(x)$ at the alcohol-free equilibrium $E_{0}$ are, respectively,

$$
D F\left(E_{0}\right)=\left(\begin{array}{cc}
F_{3 \times 3} & 0 \\
0 & 0
\end{array}\right), \quad D \mho\left(E_{0}\right)=\left(\begin{array}{cccc} 
& V_{3 \times 3} & & 0 \\
\beta & \beta & 0 & \mu
\end{array}\right)
$$

where

$$
F=\left(\begin{array}{ccc}
(1-p) \beta & (1-p) \gamma & 0 \\
p \beta & p \gamma & 0 \\
0 & 0 & 0
\end{array}\right), \quad V=\left(\begin{array}{ccc}
\alpha+\mu & 0 & 0 \\
-\alpha & \pi+\mu & -\eta \\
0 & -\pi & \eta+\mu
\end{array}\right)
$$

The model reproduction number, denoted by $R_{0}$, is thus given by

$$
R_{0}=\rho\left(F V^{-1}\right)=\frac{(1-p) \beta \mu(\pi+\eta+\mu)+(1-p) \gamma \alpha(\eta+\mu)+p \gamma(\alpha+\mu)(\eta+\mu)}{\mu(\alpha+\mu)(\pi+\eta+\mu)} .
$$

Following Theorem 2 of [18], we have the following result on the local stability of $E_{0}$.

Theorem 3.1. The alcohol-free equilibrium $E_{0}$ is locally asymptotically stable for $R_{0}<1$ and unstable otherwise.

\subsection{Global Stability of $E_{0}$}

Theorem 3.2. For system (2.4), the alcohol-free equilibrium $E_{0}$ is globally asymptotically stable if $R_{0}<1$.

Proof. We use the comparison theorem to prove the global stability of the alcohol-free equilibrium. The rate of change of the variables $\left(a_{1}, a_{2}, r\right)$ of system (2.4) can be rewritten as

$$
\left(\begin{array}{c}
\dot{a}_{1} \\
\dot{a}_{2} \\
\dot{r}
\end{array}\right)=(F-V)\left(\begin{array}{c}
a_{1} \\
a_{2} \\
r
\end{array}\right)-(1-s)\left(\begin{array}{ccc}
(1-p) \beta & (1-p) \gamma & 0 \\
p \beta & p \gamma & 0 \\
0 & 0 & 0
\end{array}\right)\left(\begin{array}{c}
a_{1} \\
a_{2} \\
r
\end{array}\right)
$$


where $F$ and $V$ are defined in (3.5). Since $s \leq 1$ for all $t \geq 0$ in $\Omega$, then

$$
\left(\begin{array}{c}
\dot{a}_{1} \\
\dot{a}_{2} \\
\dot{r}
\end{array}\right) \leq(F-V)\left(\begin{array}{c}
a_{1} \\
a_{2} \\
r
\end{array}\right) .
$$

Since the eigenvalues of the matrix $F-V$ all have negative real parts (this comes from the local stability results in Lemma 1 in [18]), then system (2.4) is stable whenever $R_{0}<1$. So, $\left(a_{1}, a_{2}, r\right) \rightarrow(0,0,0)$ as $t \rightarrow \infty$. By the comparison theorem [19], it follows that $\left(a_{1}, a_{2}, r\right) \rightarrow(0,0,0)$ and $s \rightarrow 1$ as $t \rightarrow \infty$. The $\left(s, a_{1}, a_{2}, r\right) \rightarrow E_{0}$ as $t \rightarrow \infty$. So, $E_{0}$ is globally asymptotically stable for $R_{0}<1$.

\subsection{Alcohol-Present Equilibrium}

\subsubsection{Existence of the Alcohol-Present Equilibrium}

If $R_{0}>1$, system (2.4) has a unique alcohol-present equilibrium $E^{*}\left(s^{*}, a_{1}^{*}, a_{2}^{*}, r^{*}\right)$, where

$$
\begin{gathered}
s^{*}=\frac{1}{R_{0}}, \\
a_{1}^{*}=\frac{\mu(1-p)}{\alpha+\mu}\left(1-\frac{1}{R_{0}}\right), \\
a_{2}^{*}=\frac{p(\alpha+\mu)(\eta+\mu)+\alpha(1-p)(\eta+\mu)}{(1-p) \mu(\pi+\eta+\mu)} a_{1}^{*}, \\
r^{*}=\frac{\pi}{\eta+\mu} a_{2}^{*} .
\end{gathered}
$$

\subsubsection{Global Stability of the Alcohol-Present Equilibrium}

Theorem 3.3. If $R_{0}>1$, the alcohol-present equilibrium $E^{*}$ is globally asymptotically stable.

Proof. To study the global stability of the alcohol-present equilibrium, motivated by [20-22], we use a Lyapunov function $V$ as follows:

$$
V=x_{1}\left(s-s^{*} \ln s\right)+x_{2}\left(a_{1}-a_{1}^{*} \ln a_{1}\right)+x_{3}\left(a_{2}-a_{2}^{*} \ln a_{2}\right)+x_{4}\left(r-r^{*} \ln r\right) .
$$

Applying the identity $\mu=s^{*}\left(\beta a_{1}^{*}+\gamma a_{2}^{*}\right)+\mu s^{*}$, the derivative of $V$ is given by

$$
\begin{aligned}
\dot{V} & =x_{1}\left(1-\frac{s^{*}}{s}\right) \dot{s}+x_{2}\left(1-\frac{a_{1}^{*}}{a_{1}}\right) \dot{a}_{1}+x_{3}\left(1-\frac{a_{2}^{*}}{a_{2}}\right) \dot{a}_{2}+x_{4}\left(1-\frac{r^{*}}{r}\right) \dot{r} \\
& =x_{1}\left[\mu-s\left(\beta a_{1}+\gamma a_{2}\right)-\mu s-\mu \frac{s^{*}}{s}+s^{*}\left(\beta a_{1}+\gamma a_{2}\right)+\mu s^{*}\right]
\end{aligned}
$$




$$
\begin{aligned}
& +x_{2}\left[(1-p) s\left(\beta a_{1}+\gamma a_{2}\right)-(\alpha+\mu) a_{1}-\frac{(1-p) \beta a_{1} s a_{1}^{*}}{a_{1}}\right. \\
& \left.\quad-\frac{(1-p) \gamma a_{2} s a_{1}^{*}}{a_{1}}+(\alpha+\mu) a_{1}^{*}\right] \\
& +x_{3}\left[p s\left(\beta a_{1}+\gamma a_{2}\right)+\eta r+\alpha a_{1}-(\pi+\mu) a_{2}-\frac{p \beta a_{1} s a_{2}^{*}}{a_{2}}\right. \\
& +x_{4}\left[\pi a_{2}-(\eta+\mu) r-\frac{\pi a_{2} r^{*}}{r}+(\eta+\mu) r^{*}\right] \\
& +x_{1} \mu s^{*}\left(2-\frac{s}{s^{*}}-\frac{s^{*}}{s}\right) \\
& +\left[x_{1} s^{*}\left(\beta a_{1}^{*}+\gamma a_{2}^{*}\right)+x_{2}(\alpha+\mu) a_{1}^{*}+x_{3}(\pi+\mu) a_{2}^{*}+x_{4}(\eta+\mu) r^{*}\right] \\
& + \\
& + \\
& +
\end{aligned}
$$

The positive constants $x_{1}, x_{2}, x_{3}$, and $x_{4}$ are chosen such that the coefficients of $s a_{1}, s a_{2}, a_{1}, a_{2}$, and $r$ are equal to zero, that is,

$$
\begin{gathered}
-x_{1}+x_{2}(1-p)+x_{3} p=0 \\
x_{1} \beta s^{*}-x_{2}(\alpha+\mu)+x_{3} \alpha=0 \\
x_{1} \gamma s^{*}-x_{3}(\pi+\mu)+x_{4} \pi=0 \\
x_{3} \eta-(\eta+\mu) x_{4}=0 .
\end{gathered}
$$


From (3.12), we have

$$
\begin{gathered}
x_{1}=1, \quad x_{2}=\frac{\mu(\pi+\mu+\eta) \beta s^{*}+\alpha(\eta+\mu) \gamma s^{*}}{\mu(\alpha+\mu)(\eta+\mu+\pi)}, \\
x_{3}=\frac{(\eta+\mu) \gamma s^{*}}{\mu(\pi+\eta+\mu)}, \quad x_{4}=\frac{\eta \gamma s^{*}}{\mu(\pi+\eta+\mu)} .
\end{gathered}
$$

We regroup terms in $\dot{V}$ such that $\dot{V}=\dot{V}_{1}+\dot{V}_{2}+\dot{V}_{3}$, where

$$
\begin{gathered}
\dot{V}_{1}=\mu s^{*}\left(2-\frac{s}{s^{*}}-\frac{s^{*}}{s}\right), \\
\dot{V}_{2}=x_{1} s^{*}\left(\beta a_{1}^{*}+\gamma a_{2}^{*}\right)+x_{2}(\alpha+\mu) a_{1}^{*}+x_{3}(\pi+\mu) a_{2}^{*}+x_{4}(\eta+\mu) r^{*}, \\
\dot{V}_{3}=-\frac{x_{1}\left(s^{*}\right)^{2}\left(\beta a_{1}^{*}+\gamma a_{2}^{*}\right)}{s}-x_{2}(1-p) \beta s a_{1}^{*}-x_{3} p r s a_{2}^{*}-\frac{x_{2}(1-p) \gamma a_{2} s a_{1}^{*}}{a_{1}} \\
-\frac{x_{3} p \beta a_{1} s a_{2}^{*}}{a_{2}}-\frac{x_{3} \alpha a_{1} a_{2}^{*}}{a_{2}}-\frac{x_{3} \eta r a_{2}^{*}}{a_{2}}-\frac{x_{4} \pi a_{2} r^{*}}{r} .
\end{gathered}
$$

Using the values for $x_{1}, x_{2}, x_{3}$, and $x_{4}$ in (3.13), relations in (3.12), and the equilibrium relations,

$$
\begin{gathered}
(\alpha+p \mu)(\eta+\mu) a_{1}^{*}=(1-p) \mu(\pi+\eta+\mu) a_{2}^{*} \\
x_{3} \eta r^{*}=x_{4} \pi a_{2}^{*} .
\end{gathered}
$$

We can rewrite $\dot{V}_{2}$ as

$$
\dot{V}_{2}=2 x_{2}(1-p) \beta s^{*} a_{1}^{*}+2 x_{3} p \gamma s^{*} a_{2}^{*}+4 x_{3} p \beta s^{*} a_{1}^{*}+\frac{3(1-p) \alpha \gamma s^{*} a_{2}^{*}}{\alpha+p \mu}+2 x_{4} \pi a_{2}^{*}
$$

Similarly, we can rewrite $\dot{V}_{3}$ as

$$
\begin{aligned}
\dot{V}_{3}= & {\left[-x_{2}(1-p) \beta s a_{1}^{*}-\frac{x_{2}(1-p) \beta\left(s^{*}\right)^{2} a_{1}^{*}}{s}\right] } \\
& +\left[-x_{3} p \gamma s a_{2}^{*}-\frac{x_{3} p \gamma\left(s^{*}\right)^{2} a_{2}^{*}}{s}\right]
\end{aligned}
$$




$$
\begin{aligned}
& +\left[-y \frac{x_{2}(1-p) \gamma a_{2} s a_{1}^{*}}{a_{1}}-\frac{x_{3} \alpha a_{1} a_{2}^{*}}{a_{2}}-y \frac{x_{2}(1-p) \gamma a_{2}^{*}\left(s^{*}\right)^{2}}{s}\right] \\
& +\left[-(1-y) \frac{x_{2}(1-p) \gamma a_{2} s a_{1}^{*}}{a_{1}}-\frac{x_{3} p \beta a_{1} s a_{2}^{*}}{a_{2}}\right. \\
& \left.-(1-y) \frac{x_{2}(1-p) \gamma a_{2}^{*}\left(s^{*}\right)^{2}}{s}-\frac{x_{3} p \beta a_{1}^{*}\left(s^{*}\right)^{2}}{s}\right] \\
& +\left[-\frac{x_{3} \eta r a_{2}^{*}}{a_{2}}-\frac{x_{4} \pi a_{2} r^{*}}{r}\right],
\end{aligned}
$$

where

$$
y=\frac{\alpha}{(\alpha+p \mu) x_{2}}, \quad 1-y=\frac{p \beta s^{*}}{(\alpha+p \mu) x_{2}}
$$

Let $\dot{V}_{3}=\dot{V}_{a}+\dot{V}_{b}+\dot{V}_{c}+\dot{V}_{d}+\dot{V}_{e}$, with each term representing the expression enclosed in a pair of big square brackets. Using the arithmetic-geometric mean inequality, we obtain

$$
\begin{aligned}
\dot{V}_{a} & =-x_{2}(1-p) \beta s a_{1}^{*}-\frac{x_{2}(1-p) \beta\left(s^{*}\right)^{2} a_{1}^{*}}{s} \\
& \leq-2\left[x_{2}^{2}(1-p)^{2} \beta^{2}\left(s^{*}\right)^{2}\left(a_{1}^{*}\right)^{2}\right]^{1 / 2} \\
& =-2 x_{2}(1-p) \beta s^{*} a_{1}^{*} \\
\dot{V}_{b} & =-x_{3} p \gamma s a_{2}^{*}-\frac{x_{3} p \gamma\left(s^{*}\right)^{2} a_{2}^{*}}{s} \\
& \leq-2\left[x_{3}^{2} p^{2} \gamma^{2}\left(s^{*}\right)^{2}\left(a_{2}^{*}\right)^{2}\right]^{1 / 2}=-2 x_{3} p \gamma s^{*} a_{2}^{*}, \\
\dot{V}_{c} & =-y \frac{x_{2}(1-p) \gamma a_{2} s a_{1}^{*}}{a_{1}}-\frac{x_{3} \alpha a_{1} a_{2}^{*}}{a_{2}}-y_{2} \frac{x_{2}(1-p) \gamma a_{2}^{*}\left(s^{*}\right)^{2}}{s} \\
& \leq-3\left[x_{2}^{2}(1-p)^{2} x_{3} \alpha a_{1}^{*} y^{2}\left(s^{*}\right)^{2} \gamma^{2}\left(a_{2}^{*}\right)^{2}\right]^{1 / 3}=-\frac{3(1-p) \alpha s^{*} \gamma a_{2}^{*}}{\alpha+p \mu}, \\
\dot{V}_{d} & =-(1-y) \frac{x_{2}(1-p) \gamma a_{2} s a_{1}^{*}}{a_{1}}-\frac{x_{3} p \beta a_{1} s a_{2}^{*}}{a_{2}}-(1-y) \frac{x_{2}(1-p) \gamma a_{2}^{*}\left(s^{*}\right)^{2}}{s}-\frac{x_{3} p \beta a_{1}^{*}\left(s^{*}\right)^{2}}{s} \\
\dot{V}_{e} & \leq-\frac{x_{3} \eta r a_{2}^{*}}{a_{2}-\frac{x_{4} \pi a_{2} r^{*}}{r}} \\
& \leq-4\left[x_{3} \eta r^{*} x_{4} \pi a_{2}^{*}\right]^{1 / 2}=-2 x_{4} \pi a_{2}^{*} . \\
& \left.\leq x_{2}^{2}(1-p)^{2} p^{2} x_{3}^{2}(1-y)^{2} \gamma^{2}\left(a_{1}^{*}\right)^{2} \beta^{2}\left(a_{2}^{*}\right)^{2}\left(s^{*}\right)^{4}\right]^{1 / 4}=-4 x_{3} p \beta s^{*} a_{1}^{*},
\end{aligned}
$$


Table 1: Description and estimation of parameters.

\begin{tabular}{llcc}
\hline Parameter & Description & $\begin{array}{c}\text { Estimated } \\
\text { value }\end{array}$ & $\begin{array}{c}\text { Date } \\
\text { source }\end{array}$ \\
\hline$\mu$ & Recruitment rate of the population & $0.25 \mathrm{year}^{-1}$ & {$[15]$} \\
$\beta$ & Transmission coefficient of the nonadmitting compartment & 0.3 year $^{-1}$ & {$[15]$} \\
$\gamma$ & Transmission coefficient of the admitting compartment & $0.25 \mathrm{year}^{-1}$ & Estimate \\
$\alpha$ & The fraction of $S$ being infected by $A_{1}$ and entering $A_{2}$ & Variable $^{-1}$ & \\
$\pi$ & The fraction of $A_{2}$ going into treatment & $0.05 \mathrm{year}^{-1}$ & {$[15]$} \\
$\eta$ & The fraction of $R$ who relapse into $A_{2}$ & 0.8 year $^{-1}$ & {$[15]$} \\
$p$ & $\begin{array}{l}\text { Probability of a newly infected individual who is admitting the } \\
\text { problem }\end{array}$ & Variable & \\
$1-p$ & $\begin{array}{l}\text { Probability of a newly infected individual who is not admitting the } \\
\text { problem }\end{array}$ & Variable & \\
\hline
\end{tabular}

From (3.19), we have

$$
\dot{V}_{3} \leq-2 x_{2}(1-p) \beta s^{*} a_{1}^{*}-2 x_{3} p \gamma s^{*} a_{2}^{*}-4 x_{3} p \beta s^{*} a_{1}^{*}-\frac{3(1-p) \alpha \gamma s^{*} a_{2}^{*}}{\alpha+p \mu}-2 x_{4} \pi a_{2}^{*} .
$$

From (3.16) and (3.20), we have $\dot{V}_{2}+\dot{V}_{3} \leq 0$. Therefore, $\dot{V} \leq 0$. Furthermore, $\dot{V}=0$ if and only if $\dot{V}_{1}=0$ and $\dot{V}_{2}+\dot{V}_{3}=0$. We can show that $\dot{V}=0 \Leftrightarrow\left(s, a_{1}, a_{2}, r\right)=\left(s^{*}, a_{1}^{*}, a_{2}^{*}, r^{*}\right)$. Thus, $\dot{V}$ is a negative definite with respect to $P^{*}$. So the alcohol-present equilibrium $E^{*}$ is globally asymptotically stable.

\section{Numerical Simulation}

To illustrate the analytic results obtained above, we give some simulations using the parameter values in Table 1. Numerical results are displayed in the following figures. First, we choose $p=0.8, \alpha=0.2$; numerical simulation gives $R_{0}=0.4091<1$, then Theorem 3.2 indicates that youths do not have alcohol problems. Figure 2 confirms this conclusion. Second, we choose $p=0.1, \alpha=0.4$; numerical simulation gives $R_{0}=1.8031>1$; then Theorem 3.3 shows that alcohol problems persist. Figure 3 further validates the conclusion. Finally, we choose $\alpha=0.1$; numerical simulation gives the relation between $p$ and $R_{0}$. Figure 4 confirms that $R_{0}$ shows a decline, while the number of admitting drinkers increases. From the figures above, we find that making more people admit having alcohol problems can reduce the alcohol problems. Then, the treatment of admitting drinkers is an effective measure in alcohol problems.

\section{Discussion}

We have formulated a binge drinking model with two stages and investigated their dynamical behaviors. Depending on the basic reproduction number $R_{0}$, the steady state is either the alcohol free or the alcohol-present. By using the comparison theorem, we prove that all solutions converge to $E_{0}$ when the basic reproduction number is less than one, that is, the alcohol problems disappear eventually. By constructing the Lyapunov function, we prove 

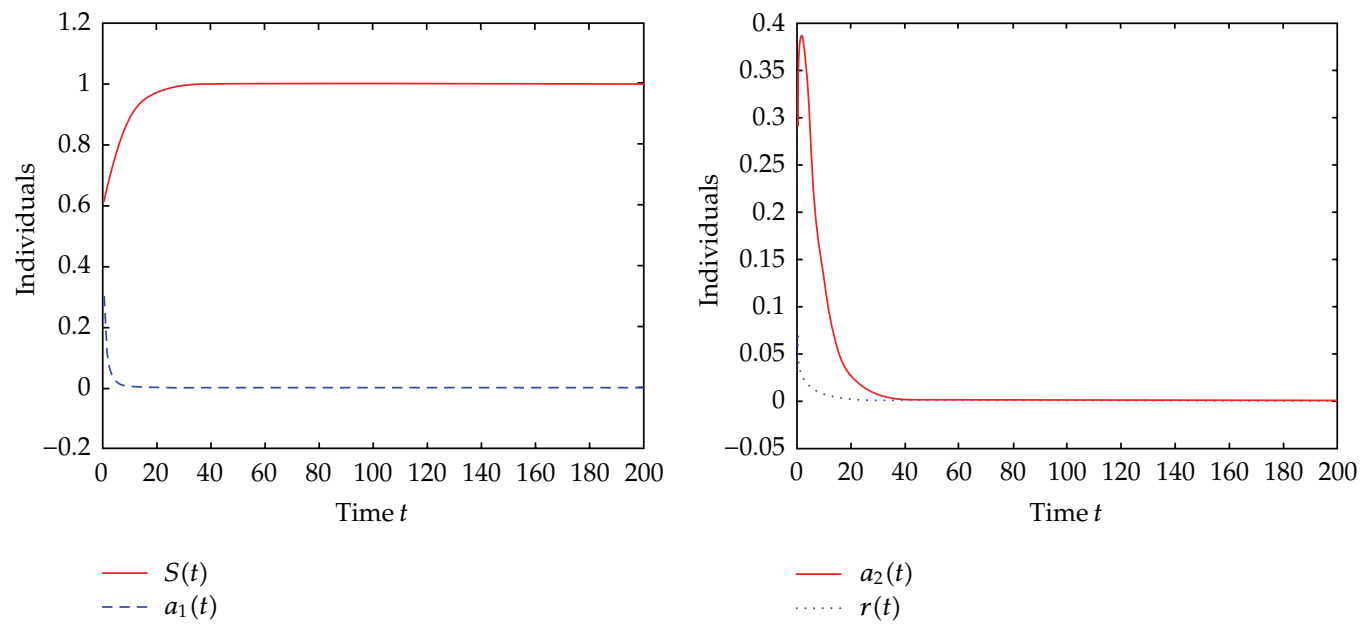

Figure 2: When $p=0.8, \alpha=0.2$, and $R_{0}=0.4091<1$, the alcohol-free equilibrium $E_{0}$ is globally asymptotically stable.
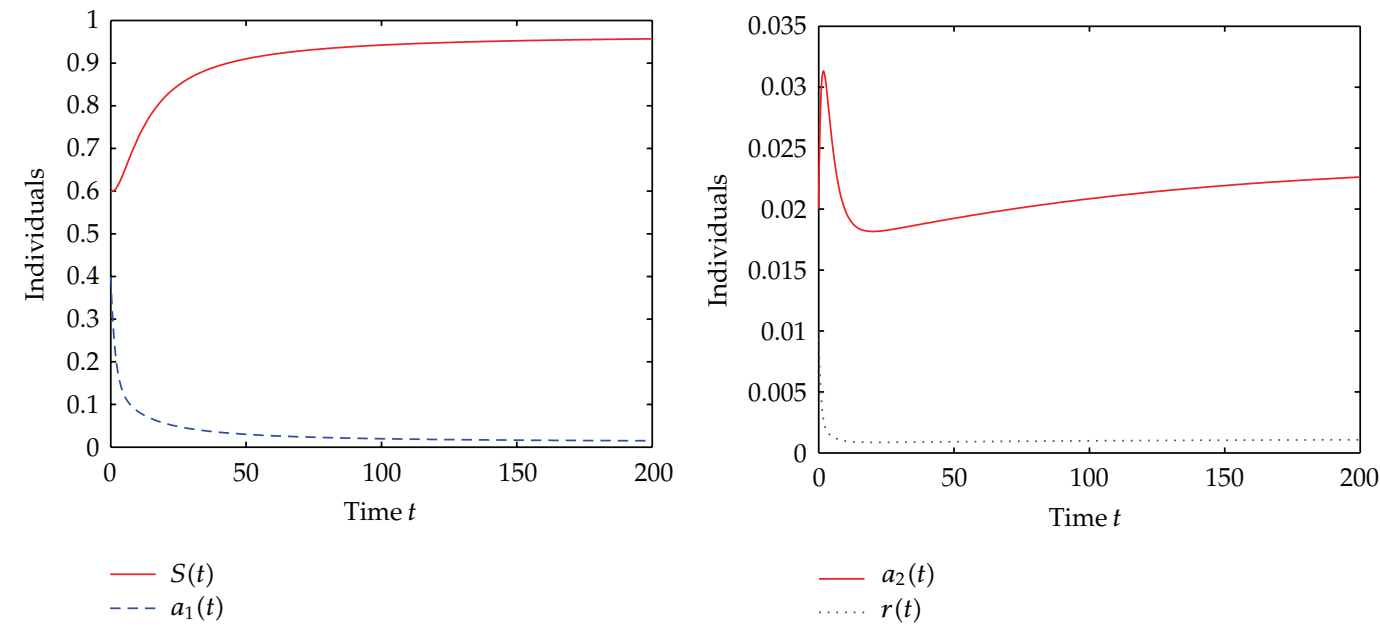

Figure 3: When $p=0.1, \alpha=0.4$, and $R_{0}=1.8031>1$, the alcohol-present equilibrium $E^{*}$ is globally asymptotically stable.

that the unique alcohol-present equilibrium is globally stable, that is, the alcohol problems will persist in the population, and the number of binge drinking individuals tends to be a positive constant when the basic reproduction number exceeds one.

To better understand the binge drinking model with two stages, we consider the more realistic model and investigate the global stability of equilibria of the model for binge drinking problem. The global stability is not discussed in the literature [15].

The basic reproductive number in this paper is given by

$$
R_{0}=\frac{(1-p) \beta \mu(\pi+\eta+\mu)+(1-p) \gamma \alpha(\eta+\mu)+p \gamma(\alpha+\mu)(\eta+\mu)}{\mu(\alpha+\mu)(\pi+\eta+\mu)} .
$$




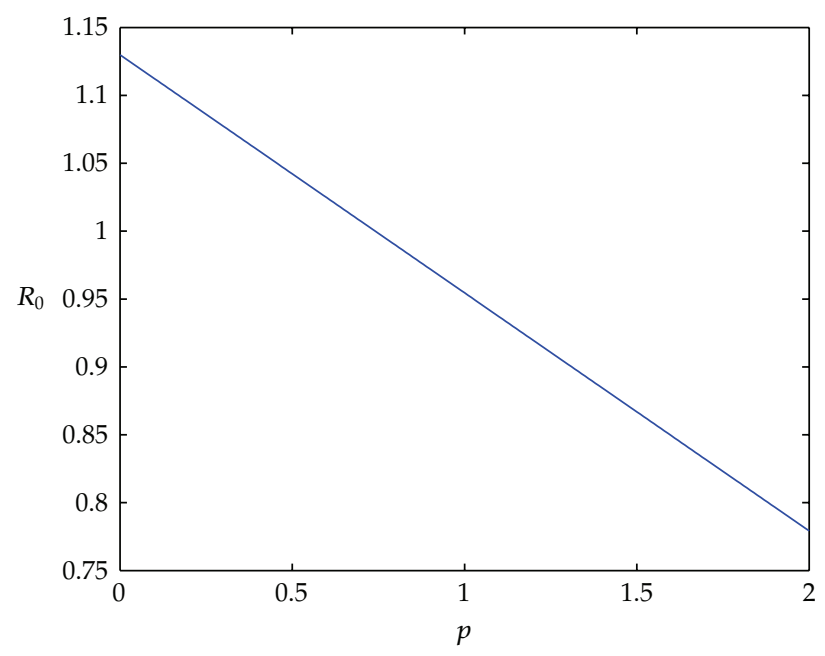

Figure 4: When $p$ increased, $R_{0}$ decline.

If the model has only one transfer from the class of susceptible individuals towards the class of nonadmitting drinkers $(p=0)$, then the basic reproductive number is

$$
R_{1}=\frac{\beta \mu(\pi+\eta+\mu)+\gamma \alpha(\eta+\mu)}{\mu(\alpha+\mu)(\pi+\eta+\mu)} .
$$

It is similar to the basic reproductive number in [15]. Comparing with the two expressions, we see that $R_{0}$ is a function on $p$. In Figure 4, we find that the basic reproductive number $R_{0}$ decreases when the number of admitting drinkers increases. In general, the nonadmitting drinkers are also unlikely to seek help. Making more people admit to have alcohol problems through the awareness programs, such as education and media programs is an effective measure to control the alcohol problems.

In deriving our model (2.2), we consider the direct transfer from the class of susceptible individuals towards the class of admitting drinkers and assume the relapse from the class of treatment individuals towards the class of admitting drinkers. However, if we hope to include another relapse from the class of treatment individuals towards the class of nonadmitting drinkers, we could modify (2.2) to the following model:

$$
\begin{gathered}
\dot{S}=\mu N-\frac{S\left(\beta A_{1}+\gamma A_{2}\right)}{N}-\mu S, \\
\dot{A}_{1}=\frac{(1-p) S\left(\beta A_{1}+\gamma A_{2}\right)}{N}+\xi R-(\alpha+\mu) A_{1}, \\
\dot{A}_{2}=\frac{p S\left(\beta A_{1}+\gamma A_{2}\right)}{N}+\eta R+\alpha A_{1}-(\pi+\mu) A_{2}, \\
\dot{R}=\pi A_{2}-(\mu+\eta+\xi) R,
\end{gathered}
$$


where $\xi$ represents relapse from the class of treatment individuals towards the class of nonadmitting drinkers.

In another way, we might consider the class of quit drinkers and also consider the following model:

$$
\begin{gathered}
\dot{S}=\mu N-\frac{S\left(\beta A_{1}+\gamma A_{2}\right)}{N}-\mu S, \\
\dot{A}_{1}=\frac{(1-p) S\left(\beta A_{1}+\gamma A_{2}\right)}{N}+\xi R-(\alpha+\mu) A_{1}, \\
\dot{A}_{2}=\frac{p S\left(\beta A_{1}+\gamma A_{2}\right)}{N}+\eta R+\alpha A_{1}-(\pi+\mu) A_{2}, \\
\dot{R}=\pi A_{2}-(\mu+\eta+\xi+\delta) R, \\
\dot{Q}=\delta R-\mu Q,
\end{gathered}
$$

where $Q(t)$ represents the population that has stopped drinking permanently at time $t$, say quit drinkers. The $\delta$ term is the rate from the class of treatment individuals towards the class of quit drinkers. We leave these works for the future.

\section{Acknowledgments}

This work was partially supported by the NNSF of China (10961018), the NSF of Gansu Province of China (1107RJZA088), the NSF for Distinguished Young Scholars of Gansu Province of China (1111RJDA003), the Special Fund for the Basic Requirements in the Research of University of Gansu Province of China, and the Development Program for HongLiu Distinguished Young Scholars in Lanzhou University of Technology.

\section{References}

[1] L. D. Johnston, P. M. OMalley, and J. G. Bachman, "National survey results on drug use from the monitoring the future study, 1975-1992," National Institute on Drug Abuse, Rockville, Md, USA, 1993.

[2] H. Wechsler, J. E. Lee, M. Kuo, and H. Lee, "College binge drinking in the 1990s: a continuing problem. Results of the Harvard School of Public Health 1999 College Alcohol Study," Journal of American College Health, vol. 48, no. 5, pp. 199-210, 2000.

[3] P. M. O'Malley and L. D. Johnston, "Epidemiology of alcohol and other drug use among American college students," Journal of Studies on Alcohol, vol. 63, no. 14, pp. 23-39, 2002.

[4] P. Ormerod and G. Wiltshire, "“"Binge" drinking in the UK: a social network phenomenon," Mind and Society, vol. 8, no. 2, pp. 135-152, 2009.

[5] H. Wechsler, J. E. Lee, T. F. Nelson, and M. Kuo, “Underage college students' drinking behavior, access to alcohol, and the influence of deterrence policies: findings from the Harvard School of Public Health College Alcohol Study," Journal of American College Health, vol. 50, no. 5, pp. 223-236, 2002.

[6] S. Mushayabasa and C. P. Bhunu, "Modelling the effects of heavy alcohol consumption on the transmission dynamics of gonorrhea," Nonlinear Dynamics, vol. 66, pp. 695-706, 2011.

[7] G. Thomas and E. M. Lungu, "The influence of heavy alcohol consumption on HIV infection and progression," Journal of Biological Systems, vol. 17, no. 4, pp. 685-712, 2009.

[8] L. Deacon, S. Hughes, K. Tocque, and M. A. Bellis, Eds., "Indications of public health in English regions. 8," Alcohol, Association of Public Health Observatories, York, UK, 2007. 
[9] G. Hay, M. Gannon, J. MacDougall, T. Millar, C. Eastwood, and N. McKeganey, "Local and national estimates of the prevalence of opiate use and/or crack cocaine use," in Measuring Different Aspects of Problem Drug Use: Methodological Developments, N. Singleton, R. Murray, and L. Tinsley, Eds., Online report OLR 16/06, London, UK, 2006.

[10] M. S. Goldman, G. M. Boyd, and V. Faden, "College drinking, what is it, and what to do about it: a review of the state of the science," Journal of Studies for Alcohol, vol. 14, pp. 1-250, 2002.

[11] F. Sanchez, X. H. Wang, C. Castillo-Chavez, D. M. Gorman, and P. J. Gruenewald, "Drinking as an epidemica simple mathematical model with recovery and relapse," in Therapists Guide to EvidenceBased Relapse Prevention: Practical Resources for the Mental Health Professional, K. A. Witkiewitz and G. A. Marlatt, Eds., pp. 353-368, Academic Press, Burlington, Vt, USA, 2007.

[12] J. L. Manthey, A. Y. Aidoo, and K. Y. Ward, "Campus drinking: an epidemiological model," Journal of Biological Dynamics, vol. 2, no. 3, pp. 346-356, 2008.

[13] A. Cintron-Arias, F. Sanchez, X. H. Wang, C. Castillo-Chavez, D. M. Gorman, and P. J. Gruenewald, "The role of nonlinear relapse on contagion amongst drinking communities," in Mathematical and Statistical Estimation Approaches in Epidemiology, pp. 343-360, Springer, New York, NY, USA, 2009.

[14] A. Mubayi, P. E. Greenwood, C. Castillo-Chávez, P. J. Gruenewald, and D. M. Gorman, "The impact of relative residence times on the distribution of heavy drinkers in highly distinct environments," Socio-Economic Planning Sciences, vol. 44, no. 1, pp. 45-56, 2010.

[15] G. Mulone and B. Straughan, "Modeling binge drinking," International Journal of Biomathematics, vol. 5, no. 1, Article ID 1250005, 14 pages, 2012.

[16] S. Reinberg, "Third of Americans Have Alcohol Problems at Some Point," 2007, http://www.washingtonpost.com/wp-dyn/content/article/2007/07/02/AR2007070201237.html.

[17] H. W. Hethcote, "The mathematics of infectious diseases," SIAM Review, vol. 42, no. 4, pp. 599-653, 2000.

[18] P. van den Driessche and J. Watmough, "Reproduction numbers and sub-threshold endemic equilibria for compartmental models of disease transmission," Mathematical Biosciences, vol. 180, pp. 29$48,2002$.

[19] V. Lakshmikantham, S. Leela, and A. A. Martynyuk, Stability Analysis of Nonlinear Systems, vol. 125, Marcel Dekker, New York, Ny, USA, 1989.

[20] X. Tian and R. Xu, "Stability analysis of a delayed SIR epidemic model with stage structure and nonlinear incidence," Discrete Dynamics in Nature and Society, vol. 2009, Article ID 979217, 17 pages, 2009.

[21] S. Yuan and B. Li, "Global dynamics of an epidemic model with a ratio-dependent nonlinear incidence rate," Discrete Dynamics in Nature and Society, vol. 2009, Article ID 609306, 13 pages, 2009.

[22] J. Li, Y. Yang, and Y. Zhou, "Global stability of an epidemic model with latent stage and vaccination," Nonlinear Analysis. Real World Applications, vol. 12, no. 4, pp. 2163-2173, 2011. 


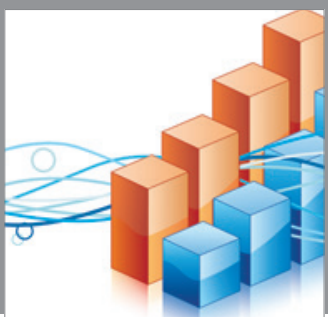

Advances in

Operations Research

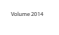

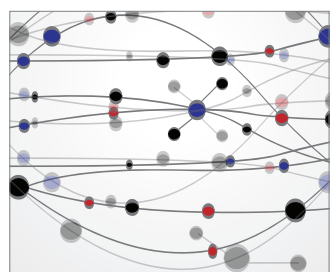

\section{The Scientific} World Journal
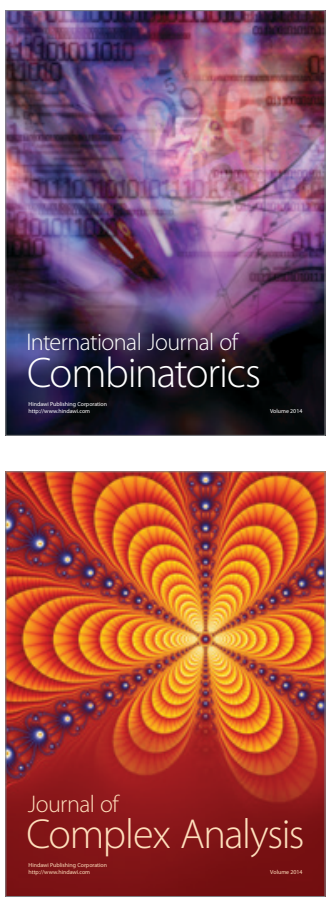

International Journal of

Mathematics and

Mathematical

Sciences
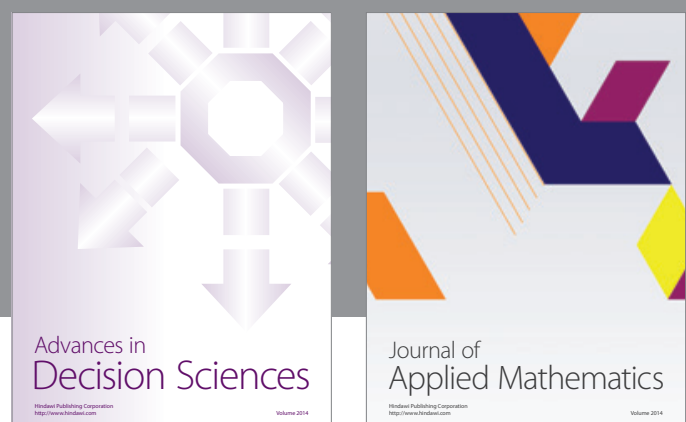

Journal of

Applied Mathematics
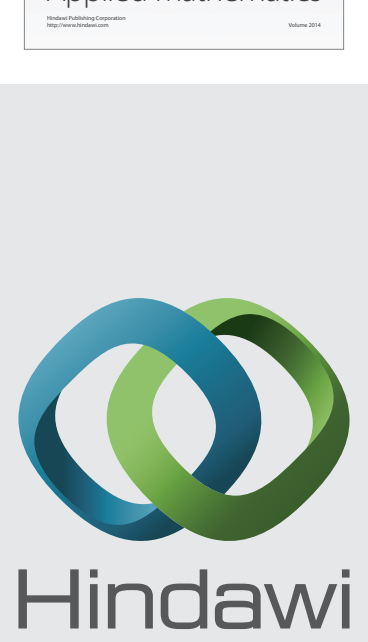

Submit your manuscripts at http://www.hindawi.com
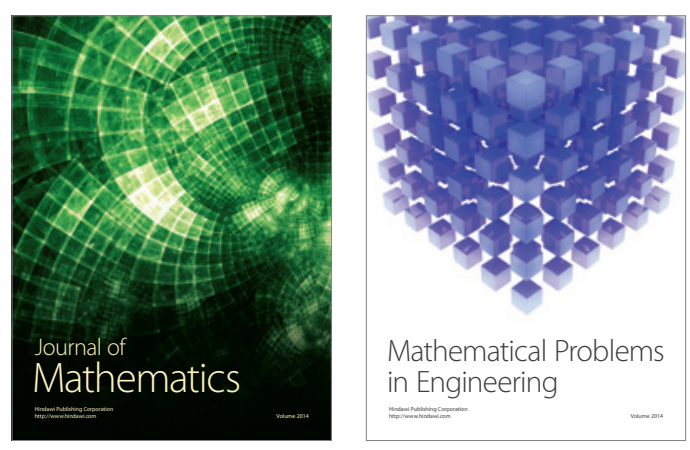

Mathematical Problems in Engineering
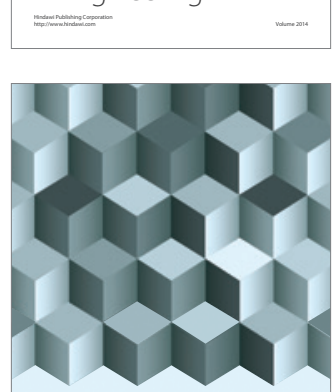

Journal of

Function Spaces
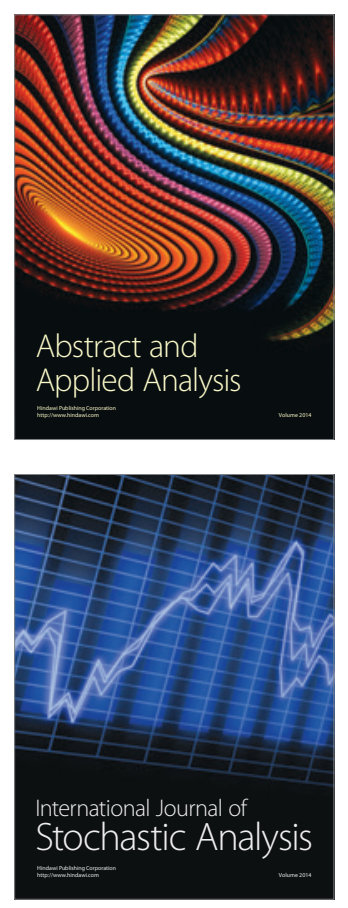

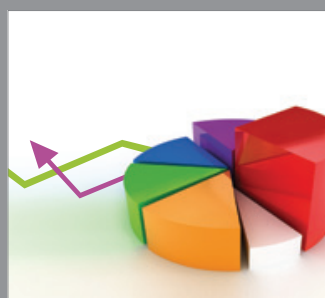

ournal of

Probability and Statistics

Promensencen
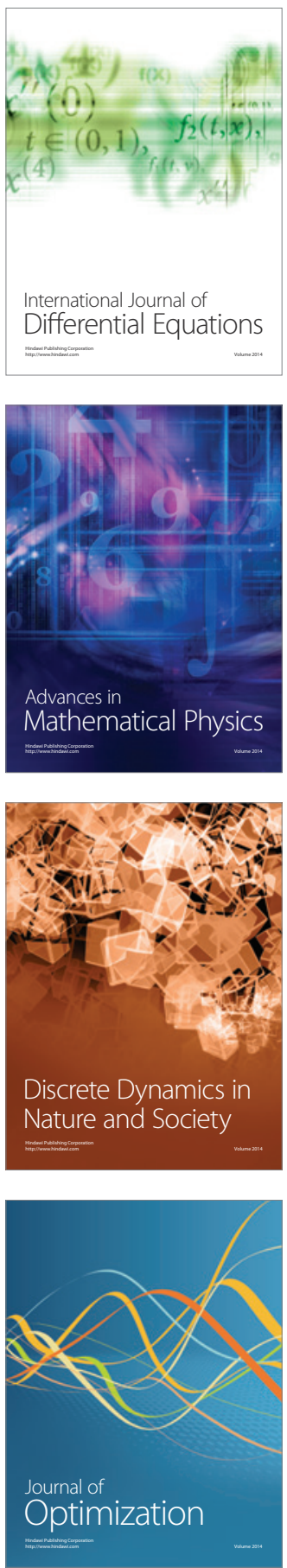